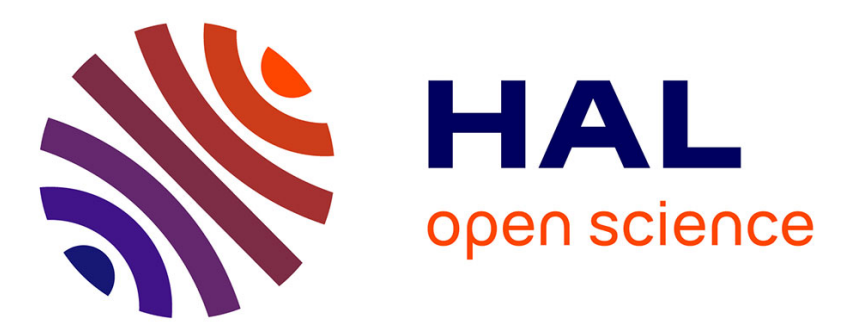

\title{
Multi-experiment state-space identification of coupled magnetic and kinetic parameters in tokamak plasmas
}

Bojan Mavkov, Emmanuel Witrant, Christophe Prieur, Didier Moreau

\section{To cite this version:}

Bojan Mavkov, Emmanuel Witrant, Christophe Prieur, Didier Moreau. Multi-experiment state-space identification of coupled magnetic and kinetic parameters in tokamak plasmas. Control Engineering Practice, 2017, 60, pp.28-38. 10.1016/j.conengprac.2016.12.006 . hal-01448484

\author{
HAL Id: hal-01448484 \\ https://hal.science/hal-01448484
}

Submitted on 28 Jan 2017

HAL is a multi-disciplinary open access archive for the deposit and dissemination of scientific research documents, whether they are published or not. The documents may come from teaching and research institutions in France or abroad, or from public or private research centers.
L'archive ouverte pluridisciplinaire HAL, est destinée au dépôt et à la diffusion de documents scientifiques de niveau recherche, publiés ou non, émanant des établissements d'enseignement et de recherche français ou étrangers, des laboratoires publics ou privés. 


\title{
Multi-experiment state-space identification of coupled magnetic and kinetic parameters in tokamak plasmas
}

\author{
B. Mavkov ${ }^{1}$, E. Witrant ${ }^{1}$, C. Prieur $^{1}$, D. Moreau ${ }^{2}$ \\ ${ }^{1}$ Univ. Grenoble Alpes, CNRS, GIPSA-lab, F-38000 Grenoble, France \\ ${ }^{2}$ CEA, IRFM, F-13108 Saint Paul-Lez-Durance, France
}

\begin{abstract}
This paper describes an identification technique for control-oriented linear time-invariant models of the coupled dynamics of the electron temperature and the poloidal magnetic flux for advanced operational tokamak scenarios. The actuators consist of two neutral beam injectors, an electron cyclotron current drive and the ohmic coil that provides the loop voltage at the plasma surface. The model is identified using a combination of subspace and output-error methods for state-space multiple-input and multiple-output system identification. This identification is applied on sets of simulated data from the METIS tokamak simulator with parameters typical of the DIII-D tokamak, and the results of the identification are presented.
\end{abstract}

Keywords: System identification, MIMO systems, Tokamak plasmas, Plasma control, Current profile control

\section{Introduction}

The tokamak is a magnetic confinement device used to heat and confine a Deuterium-Tritium plasma. The aim of tokamak research is to build reliable power production system using thermonuclear fusion [1]. There exists a number of currently operational tokamak devices as JET, DIII-D, TCV and JT-60U that are used for experimental research.

In the past years, one of the main challenges for research in controlled fusion has been the development of advanced tokamak operational scenarios to design economically attractive steady-state reactors. The heating of the tokamak machine comes from the electric currents obtained from several sources. The main sources of current in a tokamak are the ohmic coils and the external heating systems. A schematic of the coils and the magnetic fields in a tokamak is shown in Fig. 1. There are several plasma parameters, such as the safety factor, magnetic flux, electron and ion temperatures, plasma rotational velocity, etc., that define the plasma state. A key issue for advanced tokamak scenarios is

Email addresses: bojan.mavkov@gipsa-lab.fr (B. Mavkov $\left.{ }^{1}\right)$, emmanuel.witrant@ujf-grenoble.fr (E. Witrant $\left.{ }^{1}\right)$, christophe.prieur@gipsa-lab.fr (C. Prieur $\left.{ }^{1}\right)$, didier.moreau@cea.fr (D. Moreau ${ }^{2}$ ) the simultaneous control, in real time, of several plasma parameter profiles. The shape of the safety factor profile is important both for plasma thermal transport and magnetohydrodynamics (MHD) stability, and the electron temperature profile determines the plasma resistivity that governs the evolution of the safety factor profile. Building appropriate control-oriented dynamical models for the coupled evolution of these profiles is therefore important to design model-based controllers for high-performance steady state tokamak scenarios. The safety factor is related to the poloidal magnetic flux. The dynamics of the poloidal magnetic flux profile can be represented by a resistive diffusion, a parabolic equation with spatially distributed rapidly time-varying coefficients. Model-based methods for feedback control of the safety factor profile (also called the q-profile) using Multiple-Input and Multiple-Output (MIMO) finite dimensional systems are developed in [2, 3, 4, 5], and using control algorithms based on infinite dimensional control theory are developed in $[6,7,8,9]$.

The poloidal magnetic flux and the electron temperature are known to be highly coupled [1]. The heat transport equation can be presented as a one dimensional linear non-homogeneous partial differential equation with time-varying distributed diffusion coefficient and source term. The coefficients in this equation are not well known and there is no consensus about their mathemati- 

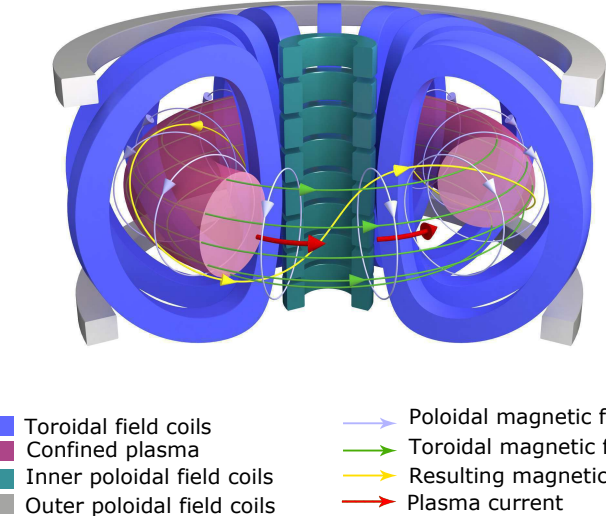

$\longrightarrow$ Poloidal magnetic field

$\longrightarrow$ Toroidal magnetic field $\longrightarrow$ Resulting magnetic field $\longrightarrow$ Plasma current

Figure 1: Representation of the coils and magnetic fields in a tokamak.

cal formulation. Only some empirical models are developed for these coefficients. An estimation of the thermal diffusion and of the source term of the heat transport model is presented in [10] and simplified model for the temperature profile obtained by using neural networks is presented in [11].

Several works have been dedicated to the integrated control of the magnetic and kinetic parameters in tokamak plasmas. A simple control algorithm based on the singular value decomposition of the experimentally deduced linear static response model was used in [4]. Then, in order to cope with fast kinetic dynamics, a two-time-scale dynamic plasma model was built by performing system identification with experimental data $[12,13]$. Such a dynamic model is based on the theory of singular perturbations and has two components. First is the slow components that consists of the dynamics of the magnetic parameters (poloidal magnetic flux, safety factor) and the slow part of the kinetic parameters (plasma density, temperature, toroidal velocity). The fast component consists of the fast dynamics of the kinetic parameters. This linearized identified model is used to control advanced tokamak scenarios in [14]. A method for profile control of the electron temperature and the safety factor based on a real-time estimation of linearized static plasma profiles is explored in [15]. First principle model-based control for the current profile and the electron temperature profile is used in [16] and physics-based control of the plasma safety factor profile and stored energy is used in $[17,18]$.

Approximate nonlinear physics-based models of the plasma dynamic evolution are being used in several simulators like CRONOS [19, 20], RAPTOR [21, 22], ASTRA [23]. These simulators are complex computer codes that have been developed to model the plasma dynamics and predict the evolution of the plasma. Some of the parameters in these simulators are calculated from first principles, but others like the heat diffusivity, are empirically or semi-empirically estimated. For example, in [24] a nonlinear least squares optimization method is used for automated parameter identification in RAPTOR, where the model parameters for the electron heat diffusivity and the electrical conductivity are estimated based on experimental data. In RAPTOR simulator it is also possible to obtain linear models directly from a linearisation of the nonlinear models around a trajectory or operating points. The linear model is obtained by calculating the Jacobian matrices computed by complex predictive RAPTOR simulation, while in this paper identification techniques are used to obtain the model.

Building a linear model of the coupled evolution of the q-profile and the electron temperature profile that can be used in a real-time control algorithm with minimum CPU time is the main goal of this paper. Using the system identification approach, this model can be obtained using only measurements of the inputs and outputs. Thus, an exact knowledge of the physics of the system is not required. In comparison with the nonlinear models used in plasma simulators, the model obtained by system identification is linear and fast, and does not require an accurate knowledge of all the parameters that would be required in a first-principle model. The identified system is obtained in state-space form, the most suitable for control design. The simplicity and generality of the system identification approach makes the technique easily adaptable to other tokamak machines with different parameters and different inputs. The identified model represents the dynamics of the kinetic and the magnetic states of the plasma combined in one system. The main goal of this kind of representation is to get a system that can be used for the simultaneous control of various parameters.

The linearized model for the evolution of kinetic and magnetic parameters in tokamaks implies a complex MIMO system. It contains several inputs such as the heating and current drive actuators (H\&CD), and several outputs represented by the Galerkin coefficients of the kinetic and magnetic profiles at discrete points of the plasma radius.

In $[12,13,14]$, the structure of the identified models was based on a singular perturbation approxima- 
tion that took advantage of the large ratio between the time scales involved in the magnetic and kinetic diffusion processes. Such two-time-scale models consist of a slow model and a fast model, with their respective sets of eigenvalues and eigenfunctions that were identified separately using an output-error identification scheme. In the present work the two-time-scale approximation is not used, so the system is more complex and could, in principle, be more accurate. In order to identify a large set of eigenfunctions that could lead to improved accuracy with respect to the two-time-scale models, a combination of a subspace identification method and outputerror identification methods is used here. The subspace identification is a powerful method for state-space identification of MIMO systems [25, 26, 27, 28]. It can be combined with the least square output-error method to get a more accurate estimation of the model [29, 30, 31]. This method can also be used for the estimation of the safety factor, which is closely related to the magnetic parameters.

The identification is performed using data provided by the METIS code, a fast integrated tokamak simulation tool for the CRONOS suite [20]. The METIS code is designed as a fast tokamak simulator implemented in MATLAB ${ }^{\circledR}$. A multiple experimental data sets can be generated using the plasma simulator. These data sets are simulating plasma discharges and the identification method can be tested. In this work a nonlinear simulator is used to obtain the plasma evolution in DIII-D tokamak.

The paper is organized as follows. The problem statements and overview of the identification procedure are elaborated in Section 2. The pre-processing of the data for the system identification is presented in Section 3. The subspace identification method is presented in Sections 4. In Section 5 the output-error method is presented along with the estimation of the reference state. Section 6 presents the simulation results of the identification method and comparisons between the data simulated with the identified linear model and the original data.

\section{Problem statement and identification procedure}

\subsection{Problem statement}

One of the key parameters to analyze the plasma stability and performance is the safety factor $q(x, t)$, defined as:

$$
q(x, t)=-\frac{\partial \Phi(x, t)}{\partial \Psi(x, t)}
$$

where $\Psi$ and $\Phi$ are the poloidal and toroidal magnetic fluxes, respectively, and $x$ is a normalized spatial variable, $0 \leq x \leq 1$. Another parameter that can be used for the control is the inverse of the safety factor $\bar{\imath}(x, t)=$ $1 / q(x, t)$, which can be considered as a more natural control variable since it is proportional to the spatial derivative of the poloidal magnetic flux whose evolution is governed by a parabolic equation. The control model of the magnetic flux is given in [32]. In a cylindrically symmetric plasma column, the time evolution of the poloidal magnetic flux is given in normalized cylindrical coordinates in [33]. In tokamaks, the poloidal flux does not reach a stationary value even when the loop voltage and the auxiliary power are kept constant. In fact, at the plasma edge, we have $V_{e x t}=-\partial \Psi(1, t) / \partial t$ and this linear flux variation induces the ohmic current in the plasma. Following [12], therefore introduce the transformation: $\Psi_{r}(x, t)=\Psi(x, t)-\Psi(1, t)$, so that the true state $\Psi_{r}(x, t)$ is the internal poloidal flux that represents the difference between the total poloidal magnetic flux and the total poloidal flux at the plasma boundary. This variable can indeed be used as a state variable of this system and is even a natural state variable of this system. It is directly related to the safety factor profile (See in Appendix) and can therefore be used for its control. The state equation for the internal poloidal flux is given by:

$$
\left\{\begin{array}{l}
\frac{\partial \Psi_{r}}{\partial t}=\frac{\eta_{\|}}{\mu_{0} a^{2}} \frac{1}{x} \frac{\partial}{\partial x}\left(x \frac{\partial \Psi_{r}}{\partial x}\right)+\eta_{\|} R_{0} j_{n i}+V_{e x t} \\
\frac{\partial \Psi_{r}}{\partial x}(0, t)=0, \quad \Psi_{r}(1, t)=0
\end{array}\right.
$$

where $R_{0}$ is the major radius of the plasma (assumed constant in time), $\mu_{0}$ is the permeability of vacuum, $\eta_{\|}$ is the parallel electrical resistivity of the plasma, $V_{\text {ext }}(t)$ is the plasma surface loop voltage, and $j_{n i}(x, t)$ is the non-inductive current-density. This equation has a stable dynamics and it can be linearized and used for the system identification procedure.

The non-inductive current density is obtained by combining the auxiliary heating and current drive sources (neutral beams, radiofrequency or electron cyclotron waves, etc.) and the bootstrap current:

$$
j_{n i}(x, t)=j_{a u x}(x, t)+j_{b s}(x, t)
$$

The dynamics of the magnetic parameters is known to be coupled with the temperature of the electrons $T_{e}$ through the plasma resistivity and the bootstrap current, both being highly dependent on the temperature. This makes the dynamics of this system highly nonlinear and coupled. In the same cylindrical approximation as for Equation (2), the time evolution of the electron temper- 
ature is given by [34]

$$
\left\{\begin{array}{l}
\frac{3}{2} n_{e} \frac{\partial T_{e}}{\partial t}=\frac{1}{a^{2}} \frac{1}{x} \frac{\partial}{\partial x}\left(x n_{e} \chi_{e} \frac{\partial T_{e}}{\partial x}\right)+Q_{e} \\
\frac{\partial T_{e}}{\partial x}(0, t)=0, \quad T_{e}(1,0)=T_{e, \text { edge }}
\end{array}\right.
$$

where $\chi_{e}$ is the electron heat diffusivity, $n_{e}$ is the electron density, and $Q_{e}$ is the total electron heating power density. The electron heating energy source (the algebraic difference between the supplied and lost energies) is calculated as a sum of several contributions. The main source of the electron heating comes from the auxiliary heating sources. Transport coefficients depend on many plasma parameters (temperature, q, etc.) as well as their radial derivative (e.g. temperature gradients, magnetic shear, etc.). These dependencies are complex and not fully known. In most of the cases empirical models must be used based on experimental data.

The identified model represents the dynamics of the variations, $\tilde{\Psi}_{r}(x, t)$ and $\tilde{T}_{e}(x, t)$ of $\Psi_{r}(x, t)$ and $T_{e}(x, t)$ around the reference values, $\bar{\Psi}_{r}$ and $\bar{T}_{e}$, subject to variations $\tilde{P}(t)$ and $\tilde{V}_{\text {ext }}(t)$ of $P(t)$ and $V_{\text {ext }}(t)$ around the reference values $\bar{P}$ and $\bar{V}_{\text {ext }}$.

The control input $P(t)$ represents the power of the heating and current drive systems e.g.:

$$
P(t)=\left[\begin{array}{l}
P_{N B I 1}(t) \\
P_{N B I 2}(t) \\
P_{E C C D}(t)
\end{array}\right]
$$

The first actuators are two deuterium neutral beam injectors: an off-axis co-current NBI power $P_{N B I 1}$ and an on-axis co-current NBI power $P_{N B I 2}$. The other two inputs are the power of electron cyclotron current drive system (ECCD) $P_{E C C D}$ and the loop voltage at the plasma surface $V_{\text {ext }}(t)$.

The output data of METIS simulator are interpolated on a unique radial grid for each parameter profile through a cubic-splines Galerkin approximation to represent the finite developments ( see [12]) as:

$$
\begin{gathered}
\Psi_{r}(x, t)=\sum_{k=1}^{n_{\Psi}} \Psi_{r k}(t) a_{k}(x) \\
T_{e}(x, t)=\sum_{k=1}^{n_{T}} T_{e k}(t) b_{k}(x)
\end{gathered}
$$

where $a_{k}$ and $b_{k}$ are cubic splines for the magnetic profile and for the temperature profile, respectively. For the spatial discretization of $\Psi_{r}(x, t), 11$ spline functions $\left(n_{\Psi}=11\right)$ were used at radial knots $x=$ $0,0.1,0.2, \ldots, 1$, and for the spatial discretization of $T_{e}(x, t), 9$ spline functions $\left(n_{T}=9\right)$ were used at radial knots $x=0,0.1,0.2, \ldots, 0.8$. The factor $1 / x$ that appears in (2) and (4) could lead to an ill defined central value due to singularity in $x=0$ when spatial discretization is applied. Spatial discretization methods dealing with systems with that kind of structure can be found in $[35,33]$.

Based on the structure of this physical system (4),(2) and flux-averaged plasma transport equations, a linearized gray-box model of the system can be postulated in the form $[12,13]$. This model can be presented in the standard state-space form by defining $\tilde{X}(t)=$ $\left[\tilde{\Psi}_{r}^{T}(t) \tilde{T}_{e}^{T}(t)\right]^{T}$ and $\tilde{U}(t)=\left[\tilde{P}^{T}(t) \tilde{V}_{e x t}(t)\right]^{T}$ :

$$
\dot{\tilde{X}}(t)=A \tilde{X}(t)+B \tilde{U}(t)
$$

with:

$$
A=\left[\begin{array}{ll}
A_{11} & A_{12} \\
A_{21} & A_{22}
\end{array}\right] \text { and } B=\left[\begin{array}{ll}
B_{P} & B_{V}
\end{array}\right]=\left[\begin{array}{cc}
B_{\Psi, P} & B_{\Psi, V} \\
B_{T_{e}, P} & 0
\end{array}\right]
$$

where $\tilde{\Psi}_{r}(t)=\left[\tilde{\Psi}_{r 1}(t), \tilde{\Psi}_{r 2}(t), . ., \tilde{\Psi}_{r n \Psi}(t)\right]^{T}$ and $\tilde{T}_{e}(t)=$ $\left[\tilde{T}_{e 1}(t), \tilde{T}_{e 2}(t), . ., \tilde{T}_{e n_{T}}(t)\right]^{T}$ are the state vectors representing the sets of the Galerkin coefficients of $\Psi_{r}(x, t)$ and $T_{e}(x, t)$, respectively. The derivation of the matrix structure of the system using a Galerkin approach applied on (2) and (4), is given in details in [12]. This model is a lumped-parameter state-space mathematical model of this physical system, with a set of state variables $\tilde{\Psi}_{r} \in \mathbb{R}^{n_{\Psi}}$ and $\tilde{T}_{e} \in \mathbb{R}^{n_{T}}$ and of inputs $\tilde{P} \in \mathbb{R}^{3}$ and $V_{\text {ext }}$, related by first-order differential equations. The matrices $A_{11} \in \mathbb{R}^{n_{\Psi} \times n_{\Psi}}, A_{12} \in \mathbb{R}^{n_{\Psi} \times n_{T}}, A_{21} \in \mathbb{R}^{n_{T} \times n_{\Psi}}$ and $A_{22} \in \mathbb{R}^{n_{T} \times n_{T}}$ are state matrices and $B_{\Psi, P} \in \mathbb{R}^{n_{\Psi} \times 3}$, $B_{\Psi, V} \in \mathbb{R}^{n_{\Psi}}$ and $B_{T_{e}, P} \in \mathbb{R}^{n_{T} \times 3}$ are input matrices to be identified. In fact, the matrix $B_{\Psi, V}$ in front of $V_{\text {ext }}$ does not need to be identified, since it is known from the definition of $\Psi_{r}$ in (2) and the derivation of the matrices structure presented in [12]. The idea for the control is to reach the desired equilibrium values of the safety factor by using only a limited number of actuators. Note that this choice of actuators can easily be modified and the identification method holds for any set of known inputs. Assuming a temporal discretization with time step $\Delta t$, at time stamps $\left[t_{1}, t_{2}, \ldots, t_{N}\right]$ where $t_{N}=t_{1}+(N-1) \Delta t$, with the corresponding discrete-time data $\left[U_{1}, U_{2}, \ldots, U_{N}\right]$ and $\left[X_{1}, X_{2}, \ldots, X_{N}\right]$ sampled from the continuous-time dynamics (8), and applying zero-order hold on the inputs, the discrete system is then obtained as

$$
\tilde{X}(t+1)=A_{d} \tilde{X}(t)+B_{d} \tilde{U}(t)
$$

where

$$
A_{d}=e^{A \Delta t}, \quad B_{d}=\int_{0}^{\Delta t} e^{A \tau} B d \tau
$$

The inverse of the safety factor can be considered as an output of the system. It depends only on the plasma pa- 
rameters and geometry, and not explicitly on the heating and current drive power. Its linearized relation with the states of the system can be represented by:

$$
\tilde{\iota}(t)=C_{\iota} \cdot\left[\begin{array}{l}
\tilde{\Psi}_{r}(t) \\
\tilde{T}_{e}(t)
\end{array}\right]
$$

The data collected for the identification were obtained from nonlinear plasma simulations, using the METIS code. METIS includes an MHD equilibrium and current diffusion solver, and combines plasma transport nonlinearity with $0-\mathrm{D}$ scaling laws and 1.5 -D ordinary differential equations. Despite its simplicity, it integrates basically all the complex features of real tokamak physics in a simplified but comprehensive and flexible way. The complexity of tokamak physics is restored through the very large number of possible options and models that the code offers for every elementary physical process (e.g. scaling laws, or fixing some source or parameter profiles and evolving others, etc.).

The simulation data were divided in two sets: one for identification and another for the validation of the identified system. For the evaluation of the MIMO system, the outputs of the simulated system were compared with the original data obtained from the non-linear METIS simulator. For each output the normalized root-meansquare error (NRMSE) fit value is calculated as:

$$
f i t_{i}(\%)=100\left(1-\frac{\left\|y_{i}(t)-\widehat{y}_{i}(t)\right\|}{\left\|y_{i}(t)-\langle y\rangle_{i}\right\|}\right) \%
$$

where $y$ is the original data, $\widehat{y}$ is the estimated outputs of the model, $\langle y\rangle$ represents the mean value of the output and $i$ represents the index of the output.

\subsection{Overview of the identification method}

Equations (8)-(12) represent a grey-box model where most of the dynamics of the system is unknown and only the value of the matrix $B_{\Psi, V}$ is known from the linearization and the discretization of (2) as shown in [12]. The identification of the lumped system is performed using the outputs represented by the Galerkin coefficients. For the identification of this state-space model a combination of two identification methods is used. First a Multivariable Output Error State sPace (MOESP) method [27] is applied. The model obtained by the MOESP method is not optimal for complex systems and when the input signals are short, but it can be used to initialize the model for the Output-Error (OE) method. A combination of subspace and iterative least-square methods has been already used in identification of MIMO state-space models [30]. The MOESP method is also very useful to determine the order of the system to be identified. The OE method initialized with the previously identified system gives a more accurate identification of the system dynamics. Before the identification, the data must be pre-processed by removing the means from the inputs and the outputs, and the original system must be transformed, through simple algebra, into a system for the zero-mean pre-processed data.

Along with the identification methods, some constraints on the eigenvalues of the system can also be introduced to reflect specific properties of the physical system. An overview of the identification cycle is presented in Fig. 2.

\section{Pre-processing the data for system identification}

The data that are used for estimation should be preprocessed by removing the offsets before the identification (e.g. see Chapter 14 in [26]). Processed data describe the relationship between the change in input signals and the change in output signals. The preprocessing operation helps to estimate more accurately linear models because the linear models identification methods cannot capture arbitrary differences between the input and output signal levels. One way of removing the offsets in the data is by removing from the system variables the reference values corresponding to steady state equilibrium around which the system has been linearized. The reference values corresponding to a given set of steady inputs could be known in the case where the so-called experimental data is obtained from nonlinear plasma simulators because the simulations could in principle be extended until the plasma reaches an equilibrium. This is not the case, however, if one uses real experimental data because, in most tokamaks, the plasma does not reach a physical equilibrium state before the end of the discharge even with steady inputs, so the measurements that can be used for system identification consist only of transient data. For the sake of generality, we shall assume that the reference values are not known a priori, and use a technique to identify them. To bring the data near the linearization point, the data are pre-processed by removing the mean values:

$$
\begin{aligned}
& \mathcal{X}(t)=X(t)-\langle X\rangle \\
& \mathcal{P}(t)=P(t)-\langle P\rangle \\
& \mathcal{V}_{\text {ext }}(t)=V_{\text {ext }}(t)-\langle V\rangle_{\text {ext }}
\end{aligned}
$$

where $\langle X\rangle=\left[\left\langle\Psi_{r}\right\rangle^{T}\left\langle T_{e}\right\rangle^{T}\right]^{T},\langle P\rangle$ and $\langle V\rangle_{\text {ext }}$ are the mean values of the measured vectors. The model corre- 
sponding to the model of the zero-mean data is:

$$
\begin{aligned}
\dot{X}(t) & =\dot{X}(t)=A(X(t)+\langle X\rangle)+B(\mathcal{U}(t)+\langle U\rangle) \\
& =A X(t)+B \mathcal{U}(t)+\Delta_{t} X
\end{aligned}
$$

where

$$
\begin{aligned}
\Delta_{t} X & =A\langle X\rangle+B\langle U\rangle \\
& =A \frac{1}{t_{N}-t_{1}} \int_{t_{1}}^{t_{N}} X(t) d t+B \frac{1}{t_{N}-t_{1}} \int_{t_{1}}^{t_{N}} U(t) d t \\
& =\frac{1}{t_{N}-t_{1}} \int_{t_{1}}^{t_{N}} \dot{X}(t) d t=\frac{X\left(t_{N}\right)-X\left(t_{1}\right)}{t_{N}-t_{1}}
\end{aligned}
$$

The term $\Delta_{t} X$ represents an offset that should be considered when the identification is performed. If the full state is measured and there is no measurement noise (as in this case), the values of $\Delta_{t} X_{j}$ are known and can be calculated for each different measurement data set $X_{j}$ represented by $\Psi_{r}(t)$ and $T_{e}(t)$.

In the black-box subspace based algorithms or in the case where there is a measurement noise in the data, this offset should be estimated considering a constant input to the system as in [36]. In such identification procedures as the output-error method, the system can be presented in a specific form where $\Delta_{t} X_{j}$ can be introduced as an additional input of the system.

\section{Subspace identification}

\subsection{MOESP method for system identification}

These simulations consist of multiple short input/output data sets. In the different reference sets, the inputs of the MIMO system are modulated in order to have a better estimation of the dynamics of the system for each input/output channel.

Thus, linear-multivariable system identification techniques are used where the multi-experiment data are merged together for one identification cycle. Techniques for the multi-experiment case are explained in $[37,31]$. Here the extension is done in a similar way for the MOESP method. The subspace method is used to find an initial system, i.e. approximations for the elements in the $A_{d}, B_{d}$ and $C_{d}$ matrices, which will be used as initial values for the recursive output-error identification. The identified system using the subspace identification method is given by the discrete-time LTI system

$$
\begin{aligned}
& \mathcal{X}(t+1)=A_{d} \mathcal{X}(t)+B_{d, e} \mathcal{U}(t) \\
& \mathcal{Y}(t)=C_{d} \mathcal{X}(t)
\end{aligned}
$$

where $A_{d} \in \mathbb{R}^{n \times n}$, where $n=n_{\Psi}+n_{T}$ is the state matrix, $B_{d, e}=\left[\begin{array}{lll}B_{d, P} & B_{d, V} & B_{d, \delta}\end{array}\right] \in \mathbb{R}^{n \times 5}$ is the input matrix and
$C_{d} \in \mathbb{R}^{n_{y} \times n}$ is the output matrix of the discrete system, where $n_{y}$ is the number of outputs. In these experiments, the input data is $\mathcal{U}(t)=\left[P^{T}(t) V_{\text {ext }}(t) 1\right]^{T}$. The output data are combined as: $\mathcal{Y}(t)=\left[\Psi_{r}^{T}(t) T_{e}^{T}(t)\right]^{T}$. Here the matrix $B_{d, \delta}$ is added to deal with the additional constant input that should identify the offset that is obtained due to the pre-processing of the data.

First, the output and input data are stored in Hankel matrices noted as $\mathcal{Y}_{1, k, N}$ and $\mathcal{U}_{1, k, N}$, respectively.

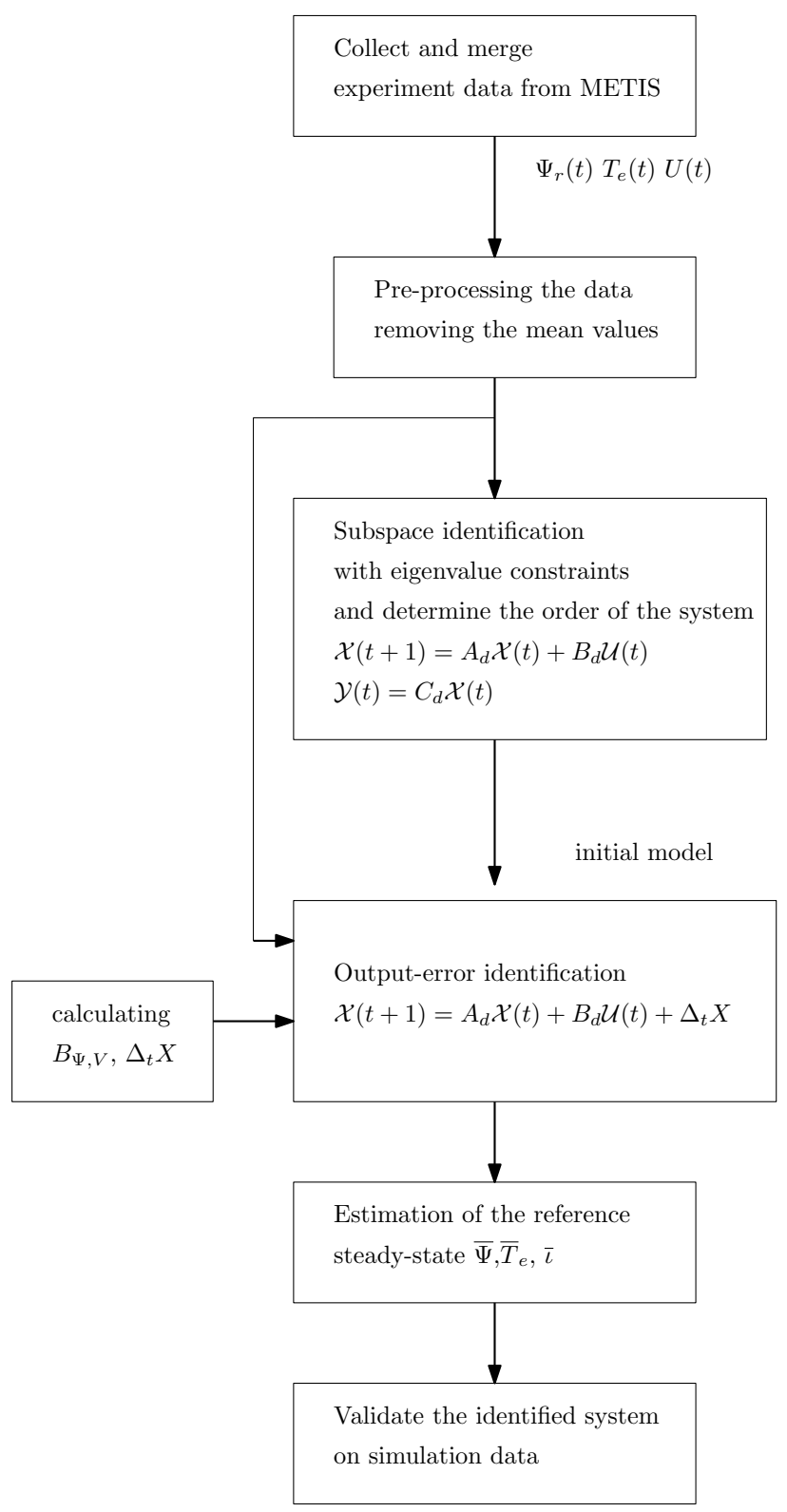

Figure 2: Overview of the method. 
The subscript 1 is the index of the first data sample and $k$ denotes the number of rows in the matrix. $N$ represents the last data sample of the experiment. The data equation in this work is extended to deal with multiple data sets that are merged together. Techniques dealing with multiple data sets in subspace identification methods are presented in [37, 31]. The LQ decomposition (where $\mathrm{L}$ is a lower triangular matrix) of the data matrix using the MOESP method for multi-experiment data is obtained as:

$$
\left[\begin{array}{c|c|c|c}
\mathcal{U}_{1, k, N_{1}}\left|\mathcal{U}_{1, k, N_{2}}\right| & \cdots & \mathcal{U}_{1, k, N_{K}} \\
\mathcal{Y}_{1, k, N_{1}}\left|\mathcal{Y}_{1, k, N_{2}}\right| & \cdots & \boldsymbol{y}_{1, k, N_{K}}
\end{array}\right]=\left[\begin{array}{cc}
L_{11} & 0 \\
L_{21} & L_{22}
\end{array}\right]\left[\begin{array}{l}
Q_{1}^{T} \\
Q_{2}^{T}
\end{array}\right]
$$

The calculation of $L_{22}$ is fully explained in [25].

\subsection{Determining the order of the system}

The identification data is extracted using the METIS code and measurement noise is not present in the data. Thus, the order of the system is estimated using the technique used for MOESP method for noise-free data [25].

By performing a singular value decomposition (SVD) on $L_{22}$, we get

$$
L_{22}=W \Sigma V^{T} \simeq\left[\begin{array}{ll}
W_{1} & W_{2}
\end{array}\right]\left[\begin{array}{cc}
\Sigma_{1} & 0 \\
0 & 0
\end{array}\right]\left[\begin{array}{c}
V_{1}^{T} \\
V_{2}^{T}
\end{array}\right]=W_{1} \Sigma_{1} V_{1}^{T}
$$

where, $\Sigma$ is a diagonal matrix with singular values of $L_{22}$ on its diagonal, the columns of $W$ are the left singular vectors and $V^{T}$ has rows that are the right singular vectors obtained by SVD. Examining the elements of the rectangular diagonal matrix $\Sigma$, a decision can be made about the choice of the order of the system. The order of the system can in principle be obtained by reducing $\Sigma$ to the first $n$ elements with highest values, $\Sigma_{1}=\operatorname{diag}\left[\sigma_{1}, \sigma_{2}, \ldots, \sigma_{n}\right]$, where $\sigma_{1} \geq \sigma_{2} \geq \ldots \geq$ $\sigma_{n}>0 \approx \sigma_{n+1} \approx \sigma_{n+2} \ldots$

In the case of this system, $\Sigma$ was calculated by taking as outputs all the 20 available measured outputs for $\Psi_{r}(x, t)$ and $T_{e}(x, t)$ at 11 and 9 radial points, respectively. The decision about the order of the system using subspace methods is heuristic. The order of the system, $n$ should be taken such that the values of the eliminated elements of $\Sigma$ are zero or close to zero. The first 10 singular values of $\Sigma$ are presented in Fig. 3.From the singular values it can be concluded that the system can be well represented if the order is taken to be $n \geq 4$. Another criterion that limits the order of the system is that the characteristic times of all the identified eigenmodes should be larger than the sampling time. In addition, the controlled system has only a few degrees of freedom because there are only 4 available actuators.
We thus restricted, for the sake of simplicity and for the needs of the control application, the order of the system and the number of controlled outputs to a maximum of $n=n_{y}=8$. The sum of the first 8 singular values is 13.23 , and it higher than $95 \%$ of the sum of all singular values in $\Sigma$. Thus, for the outputs of the system, 4 outputs were taken for the poloidal magnetic flux and 4 outputs for the electron temperature in particular radii that are important for profile control. This reduction of the number of outputs used for the identification simplifies the identification process and reduces the number of parameters that need to be estimated.

Once the order of the system has been selected, an estimate of the extended observability matrix $\Gamma_{k}$ is calculated as:

$$
\widehat{\Gamma}_{k}=W_{1} \Sigma_{1}^{1 / 2}
$$

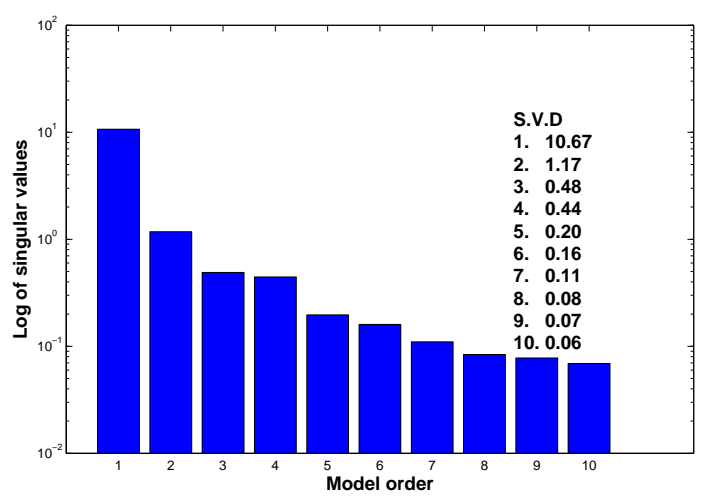

Figure 3: First 10 singular values of $\Sigma$ that indicate the order of the model.

\subsection{Eigenvalues constraints in subspace identification}

The initial estimate of $\hat{A}_{d}$ using the MOESP method is given by minimizing the cost function

$$
J_{\Gamma}\left(A_{d}\right)=\left\|\widehat{\Gamma}_{0} A_{d}-\widehat{\Gamma}_{1}\right\|_{F}
$$

where $\widehat{\Gamma}_{0}=\widehat{\Gamma}_{k}\left(1: n_{y}(k-1),:\right)$ and $\widehat{\Gamma}_{1}=\widehat{\Gamma}_{k}\left(n_{y}+1: n_{y} k,:\right)$. Here $\widehat{\Gamma}_{k}(i: j ;:)$ stands for the submatrix of $\widehat{\Gamma}_{k}$ which contains the columns from $\mathrm{i}$-th to $\mathrm{j}$-th columns. $\|\cdot\|_{F}$ denotes the Frobenius matrix norm. The solution of this linear least-squares problem with the analytic minimum is $\widehat{A}_{d}=\widehat{\Gamma}_{0}^{-1} \widehat{\Gamma}_{1}$. Some of the poles of $\widehat{A}_{d}$ obtained from (19) may be complex-conjugate, which may cause oscillations in the identified system response. This physical system is diffusive and the differential operator of the diffusion equation with diffusion coefficient is Hermitian [38], thus with real eigenvalues. Moreover the 
experimental observation does not show oscillations in the data. For these reasons we have chosen to constrain the eigenvalues in an arbitrary small band close to the real axis. As we shall see later, the systems identified within this constraint yield satisfactory simulations of the original data, so there was no real need for introducing complex-conjugate eigenvalues.

The technique of eigenvalue constraints for system identification that is used in this work is elaborated in [39]. Using this method, the eigenvalues can be constrained by defining Linear Matrix Inequalities (LMI) regions and incorporating them into the subspace identification problem. The LMI-regions define convex regions of the complex plane as LMIs.

An LMI region is a convex region $\mathcal{D}$ of the complex plane, defined in terms of a symmetric matrix $\alpha$ and a square matrix $\beta$, as:

$$
\mathcal{D}=\left\{z \in \mathbb{C}: f_{\mathcal{D}}(z) \geq 0\right\}
$$

where

$$
f_{\mathcal{D}}(z)=\alpha+\beta z+\beta^{T} \bar{z}
$$

where $\bar{z}$ is complex conjugate of $z$. The concept of using LMI regions for LMI-based synthesis is first introduced in [40]. Here we present the central theorem that is given in [40]:

Theorem 1. The eigenvalues of a matrix $\mathcal{A} \in \mathbb{R}^{n \times n}$ lie within an LMI region given by (20) if and only if there exists a matrix $N \in \mathbb{R}^{n \times n}$ such that:

$$
N=N^{T}>0, \quad \alpha \otimes N+\beta \otimes(\mathcal{A N})+\beta^{T} \otimes(\mathcal{A} N)^{T} \geq 0
$$

The concept of constraints based on LMI regions is incorporated in the subspace identification problems with the methods based on the extended observability matrix proposed by [39].

In order to get a modified model that consists of eigenvalues close to the real axis, the cost function (19) is modified as:

$$
J_{\Gamma}(M, N)=\left\|\widehat{\Gamma}_{0} A_{d} N-\widehat{\Gamma}_{1} N\right\|_{F}=\left\|\widehat{\Gamma}_{0} M-\widehat{\Gamma}_{1} N\right\|_{F}
$$

where $\mathrm{N}$ is a right-hand weighting matrix and $M=A_{d} N$. The optimization problem with convex constraints is stated as follows:

Given the estimate of the extended observability matrix $\widehat{\Gamma}$ and the LMI region described by parameters $\alpha$ and $\beta$,

$$
\begin{array}{ll} 
& \min J_{\Gamma}(M, N) \\
\text { subject to : } & \alpha \otimes N+\beta \otimes M+\beta^{T} \otimes M^{T} \geq 0 ; \\
& N=N^{T}>0
\end{array}
$$

with:

$$
\beta=\left[\begin{array}{cc}
0 & 1 \\
-1 & 0
\end{array}\right]
$$

where $\alpha=2 \delta$ is a small number that limits the imaginary part of the poles $p \in \mathbb{C}$ of the identified system into an arbitrary small band around the real axis in the complex plane represented by the set $\mathcal{R}=\{p \in \mathbb{C}:|\operatorname{Im}(p)| \leq$ $\delta, \delta \geq 0\}$.

Once $M$ and $N$ have been found, the new estimate is calculated as: $\hat{A}_{d}=M N^{-1}$. The convex optimization problem is solved using the YALMIP toolbox for MATLAB [41]. The matrices $C_{d}$ and $B_{d, e}$ were obtained using the standard MOESP method [25].

This identification method is not always sufficient for large MIMO system. As will be seen in Section 5, the model obtained by the MOESP for the problem discussed in this paper yields to some fitting errors when comparing the simulated outputs with the original data. However, this method provides a good guess for initializing the system. We have therefore used this model as a starting point for an iterative process in which the order of the system is fixed and the model matrices are optimized in each iteration by performing an output-error identification.

\section{Output-error identification}

\subsection{Estimation of the state-space matrices}

The output-error method is an iterative method [30] and requires initial values of the parameters that are estimated. The subspace identification method presented in Section 4 provides an initial model of the system. The model identified with the subspace method can be easily transformed in a form such that the output matrix $C_{d}$ is an identity matrix $C_{d}=I_{n_{y}}$. This representation of the state space model is called an observable canonical form. It can be used when direct measurement of the states $\mathcal{Y}=\mathcal{X}$ are available and pre-process the data as explained in Section 3. Also the known values of the matrices $B_{\Psi, V}$ and $\Delta_{t} X$ at this stage are introduced. Representing the system in this form avoids the need to identify the output matrix $C$, which reduces the number of parameters that needs to be estimated.

Using an iterative method the vector $\theta_{1}=$ $\left[\operatorname{vec}\left(A_{d}\right)^{T} \operatorname{vec}\left(B_{d, P}\right)^{T}\right]^{T}$ that contains all the unknown matrices $A$ and $B_{p}$ is estimated by minimizing the squared error between the measured states $X$ and the estimated $\hat{\gamma}$

$$
\min J_{K}\left(\theta_{1}\right)
$$




$$
\begin{aligned}
J_{K}\left(\theta_{1}\right)= & \frac{1}{K} \sum_{i=1}^{K} \frac{1}{N_{i}} \sum_{j=1}^{N_{i}}\left\|X_{i}\left(t_{j}\right)-\hat{X}_{i}\left(t_{j}, \theta_{1}\right)\right\|_{2}^{2} \\
& =\frac{1}{K} E_{K}\left(\theta_{1}\right)^{T} E_{K}\left(\theta_{1}\right)
\end{aligned}
$$

where:

$$
E_{K}\left(\theta_{1}\right)=\left[E_{N_{1}}^{1}\left(\theta_{1}\right)^{T} E_{N_{2}}^{2}\left(\theta_{1}\right)^{T} \cdots E_{N_{K}}^{K}\left(\theta_{1}\right)^{T}\right]^{T}
$$

and

$$
E_{N_{i}}^{i}\left(\theta_{1}\right)=\frac{1}{\sqrt{N_{i}}}\left[e^{i}(1)^{T} e^{i}(2)^{T} \cdots e^{i}\left(N_{i}\right)^{T}\right]^{T}
$$

is the error vector where $e^{i}(j)=\mathcal{X}_{i}\left(t_{j}\right)-\hat{\mathcal{X}}_{i}\left(t_{j}, \theta_{1}\right)$. As in the subspace identification approach (see Section 4), the output-error method is set for a multi-experiment data set, where experiments with different modulations of the inputs are merged for a better estimation of the dynamics of the MIMO system. In this application, the multiple-cost approach $[42,26]$ for the definition of the cost function (26) is used. For the identification, there are the measured values of the set: $\left\{\mathcal{U}_{j, i}, \mathcal{X}_{j, i}\right\}$ with $j=$ $1,2, \ldots, N_{i}$ and $i=1,2, \ldots, K(K$ denotes the number of experiments and $N_{i}$ is the number of data samples in the $i$-th experiment).

The estimated state and initial condition are given by:

$$
\begin{aligned}
& \hat{X}_{j}\left(t+1, \hat{\theta}_{1}\right)=A_{d}\left(\hat{\theta}_{1}\right) \hat{X}_{j}\left(t, \hat{\theta}_{1}\right)+B_{d, e}\left(\hat{\theta}_{1}\right) \mathcal{U}_{e}(t) \\
& \hat{X}_{j}(0)=\mathcal{X}_{j}(0)
\end{aligned}
$$

where the inputs are combined in one vector $\mathcal{U}_{e}(t)=$ $\left[\mathcal{P}^{T}(t) \mathcal{V}_{\text {ext }}(t) 1\right]^{T}$ and the matrix $B_{d, e}=\int_{0}^{\Delta t} e^{A \tau} B_{e} d \tau$ where $B_{e}=\left[B_{p} B_{v} \Delta_{t} X_{j}\right]$. The estimate (30) is presented in a discrete form with a discretization time equal to the sampling time of the experiments, $\Delta t=5 \mathrm{~ms}$.

Using the estimate in (30), the minimization of (27) can be performed by using a iterative gradient search method. In this application we use the LevenbergMarquardt method. The initial value of $\theta_{1}$ for the optimization is the one obtained previously with the MOESP method. In Fig. 4 the comparison between the outputs of the identified systems with MOESP and output error methods are presented for a particular experiment. From the plots it can be easily noticed that the results, obtained using only the MOESP subspace method, are not satisfactory due to the low NRMSE fit value of the compared outputs. Applying an additional optimization to the model, using the output-error method, improves the performance of the identified system.

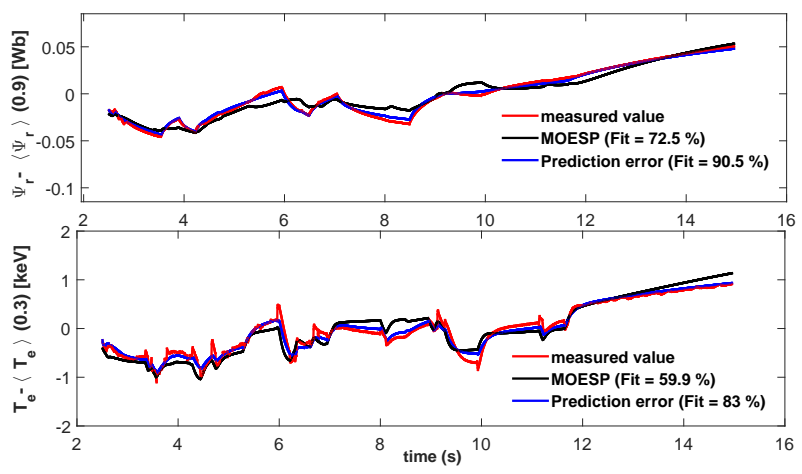

Figure 4: Comparison between the measured values of the simulated system and the outputs of the identified system for the MOESP and the output-error methods for simulation \# 22 (see Table 1).

\subsection{Estimation of the reference steady-state}

Once the best fitting model is obtained and the optimal discrete representation of the system is transformed into a continuous-time linear time invariant (LTI) model $\left[A, B, C_{l}\right]$, the reference equilibrium values of the states as in [12] could be estimated. Considering a constant input applied for a sufficiently long time, $U(t)=\bar{U}$, the steady state values $\bar{\Psi}_{r}$ and $\bar{T}_{e}$ are obtained. At steady state the plasma is in equilibrium, and $\bar{X}$ can be calculated using the steady state solution $(\dot{X}(t)=0)$ of (15), and estimating the offset by (16) as:

$$
\bar{X}=-A^{-1}\left[B \cdot(\bar{U}-\langle U\rangle)+\Delta_{t} X\right]+\langle X\rangle
$$

The reference equilibrium point for the inverse of the safety factor is then:

$$
\bar{\imath}=C_{\iota} \bar{X}
$$

With this approximate estimation of the reference states, the reduced states $\tilde{X}$ around the reference point can be found. The error of the approximation can only introduce a constant offset both on the controlled variables and their target values. It should therefore have no effect on the control action, which depends only on their difference.

\section{Identification results}

For the identification of the model, 22 data sets from the METIS tokamak simulator were provided. The plasma parameters were those of a typical DIII-D steady state scenario that is described with more details in [14]. The toroidal field is $1.8 \mathrm{~T}$, the central plasma density is 


\begin{tabular}{|c|c|c|c|c|}
\hline sim. number & $V_{\text {ext }}(V)$ & $P_{N B I 1}(M W)$ & $P_{N B I 2}(M W)$ & $P_{E C C D}(M W)$ \\
\hline 1 & 0.02 & 1.5 & 2.5 & 5 \\
\hline $2-6$ & $-0.030-0.120$ & 1.5 & 2.5 & 5 \\
\hline $7-8$ & 0.02 & 1.5 & $0-5$ & 5 \\
\hline $9-10$ & 0.02 & 1.5 & 2.5 & $2.5-7.5$ \\
\hline $11-12$ & 0.02 & $0-5$ & 2.5 & 5 \\
\hline 13 & 0.02 & 1.5 & $0-5$ & $2.5-7.5$ \\
\hline 14 & 0.02 & $0-4$ & 2.5 & $2.5-7.5$ \\
\hline $15-18$ & 0.02 & $0-4$ & $0-5$ & $2.5-7$ \\
\hline $19-20$ & $-0.23-0.27$ & $0-4$ & $0-5$ & $2.5-7.5$ \\
\hline 21 & $-0.030-0.120$ & $0-4$ & 2.5 & $2.5-7.5$ \\
\hline 22 & $-0.030-0.120$ & 1.5 & $0-5$ & $2.5-7.5$ \\
\hline
\end{tabular}

Table 1: Table of the nonlinear METIS simulations used for the system identification showing the minimum and maximum values of the squarewave modulated inputs (simulation numbers used for the identification: \# 1,2, 3, 4, 11, 14, 16, 17, 18, 21, 22).
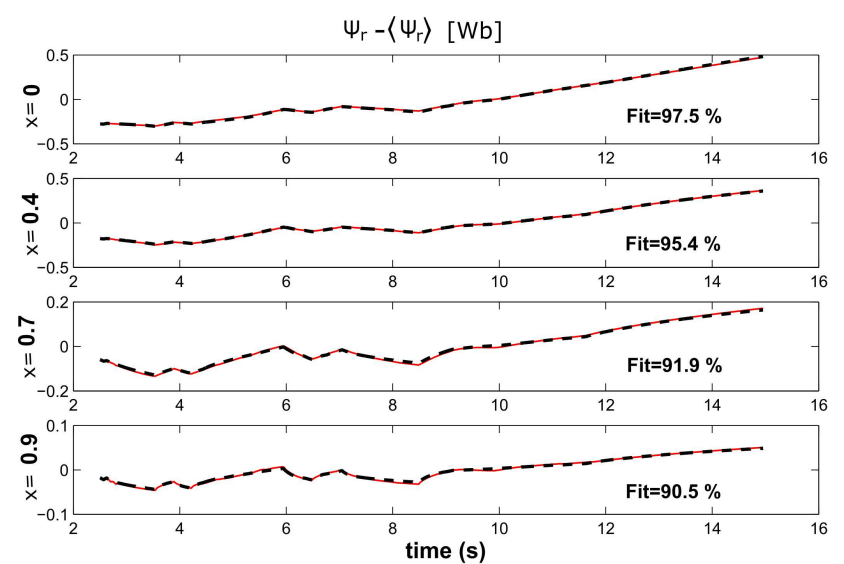

Figure 5: Plot of $\Psi_{r}(t)$ vs time for the simulation \# 22 (see Table 1), The black dashed traces represent the outputs of the simulation of the identified system and the red traces represent the outputs of the original METIS simulation. The fit parameter defined in Eq. (13) is indicated in each frame.

$5 \cdot 10^{19} \mathrm{~m}^{-3}$ and the plasma current varies between 0.6 MA and 1.2 MA depending on the values of the heating and current drive actuators. The simulations were divided into several groups presented on Table 1 . In each group, either a single input was modulated or different inputs were modulated in order to have a better estimation of the response of the system when the various inputs are simultaneously varying. The inputs were modulated using pseudorandom binary sequences in order to excite all the relevant frequencies which provides an accurate model that is valid in a large frequency range. In a real tokamak, such square wave excitation of the actuators, and in particular of $V_{\text {ext }}$, may not be possible due to the finite response time of the actuators to their
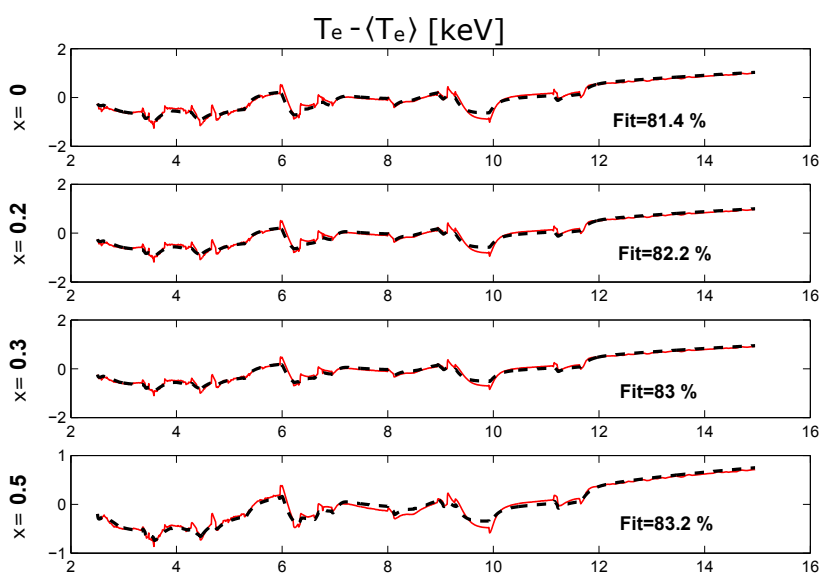

Figure 6: Plot of $T_{e}(t)$ vs time for the simulation \# 22 (see Table 1), The black dashed traces represent the outputs of the simulation of the identified system and the red traces represent the outputs of the original METIS simulation. The fit parameter defined in Eq. (13) is indicated in each frame.

command. However, the response model we are seeking here is to provide the response of the plasma to changes in the actuator commands, rather than the response to the actual input powers and surface voltage.

Half of the data set is merged to identify the model and the other half is used for the validation stage. The simulation time for each data set is $15 s$ and only the data after $2.5 s$ were used, i.e when the system outputs reach values close to the reference values around which the linear model is sought. The measurements are taken with a sampling time of $0.005 \mathrm{~s}$. The four actuators of the system, $P_{N B 1}, P_{N B 2}, P_{E C C D}$ and $V_{e x t}$ have allowed ranges of variation between $0-5 \mathrm{MW}, 2.5-10 \mathrm{MW}$, $0-4 \mathrm{MW}$ and $-0.2-0.5 \mathrm{~V}$, respectively. 


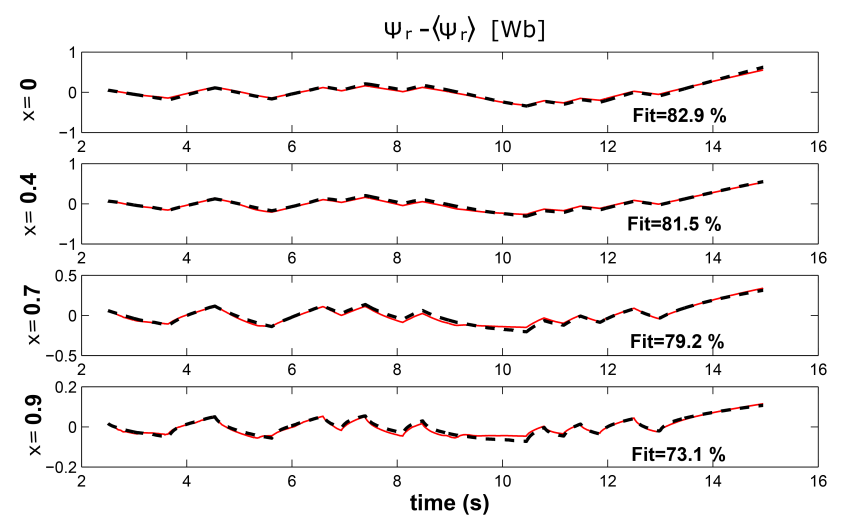

Figure 7: Plot of $\Psi_{r}(t)$ vs time for the simulation \# 19 (see Table 1), The black dashed traces represent the outputs of the simulation of the identified system and the red traces represent the outputs of the original METIS simulation. The fit parameter defined in Eq. (13) is indicated in each frame.

For the outputs of the system, 4 Galerkin coefficients were chosen from the poloidal magnetic flux profile $\Psi$ and the safety factor $\iota$, at knots $x=[0,0.4,0.7,0.9]$ and 4 Galerkin coefficients were chosen for the electron temperature, $T_{e}$ at knots $x=\left[\begin{array}{ll}0,0.2,0.3,0.5 & 0 . \text { Thus }\end{array}\right.$ the identified system is of order 8 .

The characteristic time constants of the estimated system are: $7.69 s, 1.0 s, 0.75 s, 0.62 s, 0.13 s, 0.11 s$, $0.07 s, 0.01 s$. The estimated $\widehat{A}$ satisfies the eigenvalue constraints described in Sections 4.2. Simulations were included where only specific actuators were modulated while the others are fixed for a better estimation of the columns of $B_{P}$ that are related to these inputs. The calculated value of $B_{\Psi, V}$, which is known from the physics of the problem, provides the response of the poloidal magnetic flux to the most powerful actuator in the system, $V_{\text {ext }}$.

Note that this system is a linearized model that represents the dynamics of the kinetic and magnetic profiles in a tokamak in a relatively broad vicinity of the linearization point, since the reference data set has a large variation of the actuators. Despite the highly nonlinear dynamics of the physical system, this model can be used only if the states of the system are in this broad vicinity, and therefore it is restricted to profile control applications in a particular tokamak and plasma scenario (toroidal magnetic field, plasma shape and average density) but with relatively large power variations (several megawatts). By taking the mean value of all the inputs used for the identification: $P_{N B I 1}=$ $2.3 M W, P_{N B I 2}=4.8 M W, P_{E C C D}=1.1 M W$ and $V_{\text {ext }}=0.028 \mathrm{~V}$ as reference inputs, the reference states

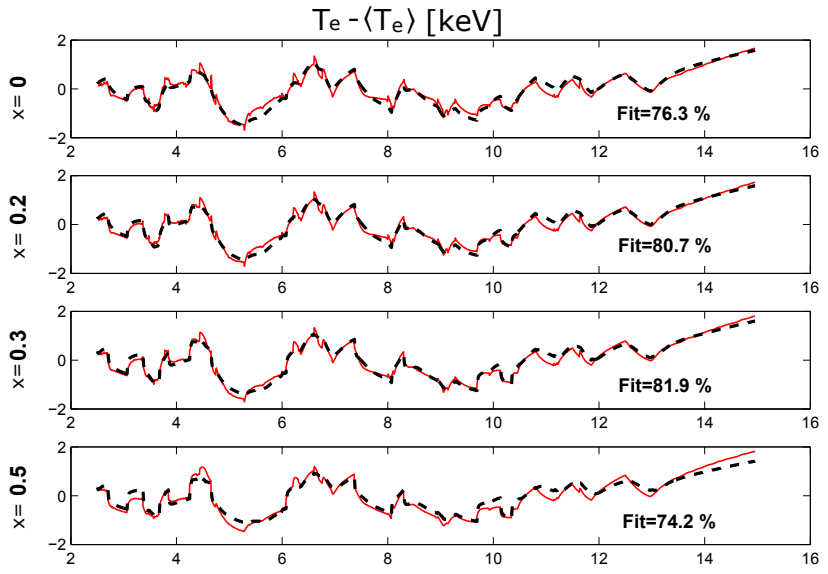

Figure 8: Plot of $T_{e}(t)$ vs time for the simulation \# 19 (see Table 1), The black dashed traces represent the outputs of the simulation of the identified system and the red traces represent the outputs of the original METIS simulation. The fit parameter defined in Eq. (13) is indicated in each frame.

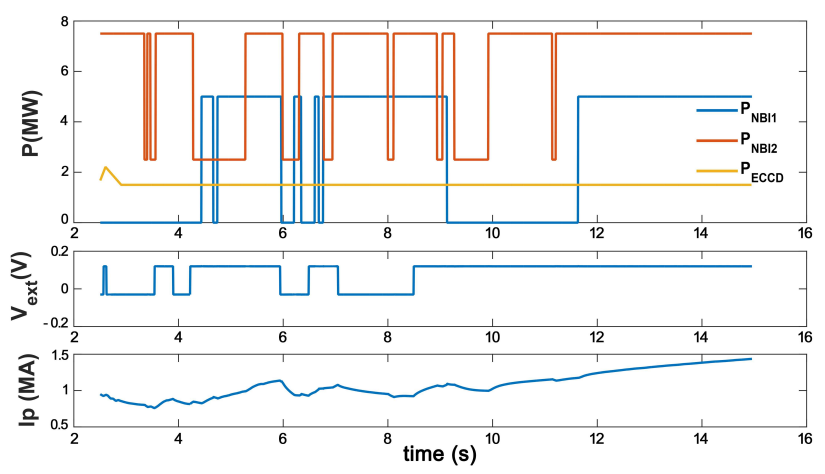

Figure 9: Plot of the four inputs $\left(P\right.$ and $\left.V_{\text {ext }}\right)$ and the total plasma current $\left(I_{p}\right)$ vs time for simulation \# 22 (see Table 1 ).

of the identified system can be calculated. Calculating the reference states for $\Psi$ and $T_{e}$ using (31), we get: $\bar{\Psi}_{r}=\left[\begin{array}{llll}2, & 1.43, & 0.61, & 0.18\end{array}\right]^{T} \mathrm{~Wb}$ and $\bar{T}_{e}=$ $[5.56,5.31,5.03,3.94]^{T} \mathrm{keV}$.

The results of the system identification can be evaluated by comparing the data predicted by the identified model with the original data. The inputs waveforms used in the simulation \# 22 that is included in the identification data are presented in Fig. 9 and the identification results in Fig. 5 and Fig. 6. The results for simulation \# 19, which is not used for identification but only for validation, are also presented. The input waveforms are presented in Fig. 10 and the evaluation results in Fig. 7 and Fig. 8. In both simulations all the inputs were modulated and the plots of the reference data (METIS 


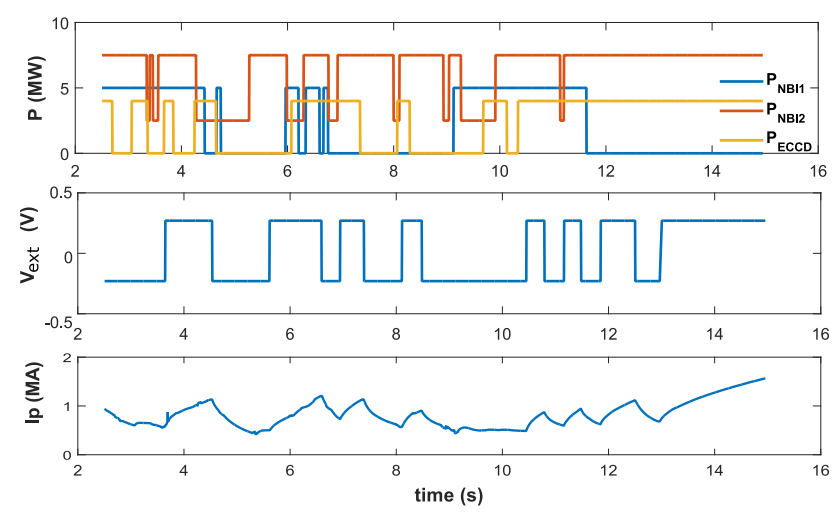

Figure 10: Plot of the four inputs $\left(P\right.$ and $\left.V_{\text {ext }}\right)$ and the total plasma current $\left(I_{p}\right)$ vs time for simulation \# 19 (see Table 1 ).

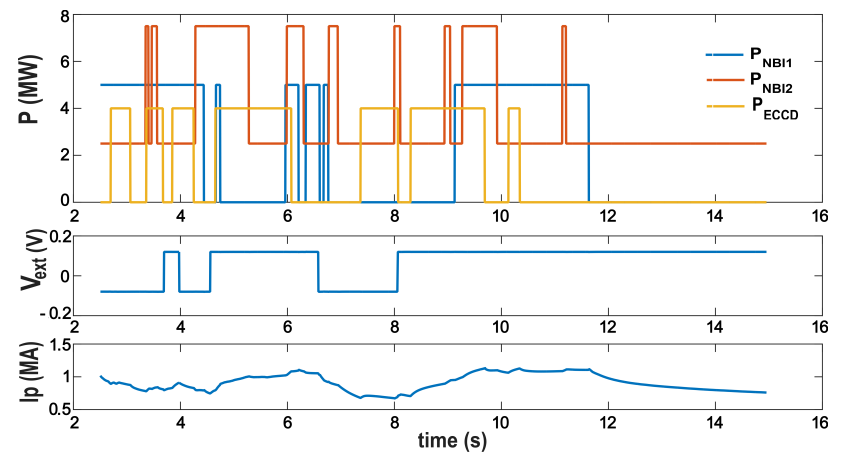

Figure 11: Plot of the inputs $\left(P\right.$ and $\left.V_{e x t}\right)$ and the total plasma current $\left(I_{p}\right)$ vs time and the plasma for simulation \# 20 (see Table 1$)$.

simulation) are compared with the output data predicted by the identified system. For each output of the system, the fit parameter values varied from about $70-98 \%$ for $\Psi_{r}(x, t)$ outputs and $60-88 \%$ for $T_{e}(x, t)$. The quality of the fit is varying within these ranges for all different simulation data except for simulations \# 15 and \# 20. The fit parameters for simulations \# 15 and \# 20 were also in the same range if the data is restricted to $t<11.6 \mathrm{~s}$, but they become poor at the end of the simulation (11 $s<t \leq 15 s$ ), yielding fit parameters around $24-45 \%$ for $\Psi_{r}(x, t)$ and $55-58 \%$ for $T_{e}(x, t)$. The results for simulation \# 20 are presented in Fig. 12 and Fig. 13 and the inputs in Fig. 11. This is explained by the fact that the total power dropped down to $2.5 \mathrm{MW}$ between $11.6 s$ and $15 s$, which results in a low temperature plasma $\left(T_{e}(0, t)<2.3 \mathrm{keV}, T_{e}(0.2, t)<2 \mathrm{keV}\right.$, $\left.T_{e}(0.3, t)<1.8 \mathrm{keV}, T_{e}(0.5, t)<1.24 \mathrm{keV}\right)$ where nonlinearities become more important. The dynamics of the temperature is faster than the dynamics of magnetic flux and when the temperature states are out of the validity domain, this implies a bad estimation of the magnetic flux states because of their strong coupling. Note that when we have short temperature drops, the estimate remains reasonably accurate. The identification results have shown that a linearized multivariable model of the coupled dynamics using a limited number of actuators can be obtained and that the model fits the original data satisfactorily when the power remains in the range of $2.5-16.5 \mathrm{MW}$.

\section{Conclusion}

This identified LTI model can be used in future work for the control of the coupled parameters in tokamaks. The identification scheme can be easily adapted to different tokamaks and in different conditions where the inputs are different than those used in this study.

The actuator variations used in METIS to obtain the identification/validation data are quite large (several megawatts, fractions of a volt) and typical of the variations that will be allowed during control experiments, with plasma current varying between 0.6 MA and 1.2 MA. As long as the toroidal field and plasma shape do not change, the identified model should then be appropriate for control applications. Otherwise, if a nonlinear model is not available, the only way to use the present approach is to perform series of linear model identifications around different plasma reference profiles. Previous approaches $[12,13,14]$ to the simultaneous control of magnetic and kinetic variables in a tokamak based on the same postulated linear system structure used singular perturbation methods (a two-timescale approximation) to divide the system into a slow and a fast system that were identified separately. In contrast, the linear model obtained here contains the whole coupled dynamics of the electron temperature and the poloidal magnetic flux, which may be more adequate for some tokamak machines, depending on the difference of the kinetic and magnetic time scales in a particular machine. The identification method presented in this paper is faster than the one presented in the previous approaches. The execution time of the subspace identification takes about 10 seconds, while the iterative outputerror methods execution time takes about 180 seconds. This combination of subspace and output-error methods could also be used within the two time scale estimation. It can provide a better estimate of the respective order of the slow and the fast models based on the information contained in the low frequency and high frequency data set, respectively. 

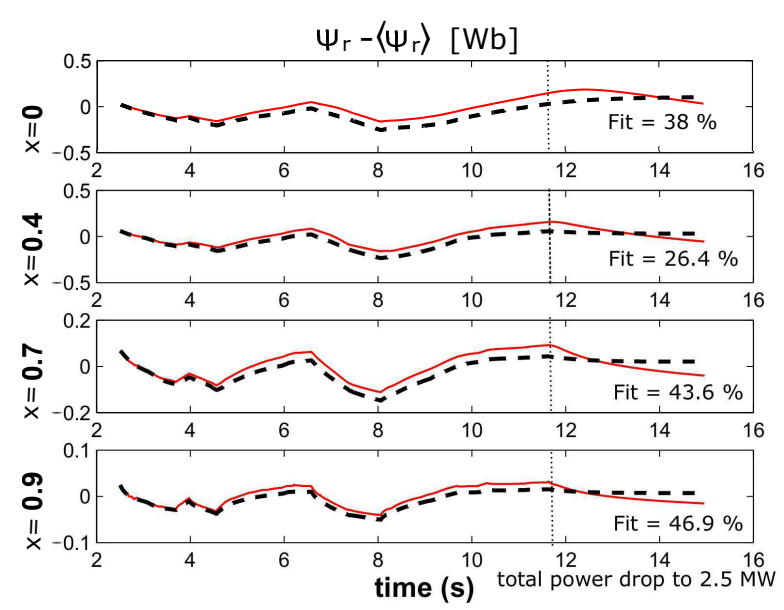

Figure 12: Plot of $\Psi_{r}(t)$ vs time for the simulation \# 20 (see Table 1), The black dashed traces represent the outputs of the simulation of the identified system and the red traces represent the outputs of the original METIS simulation. The fit parameter defined in Eq. (13) is indicated in each frame. In this simulation at $11.6 \mathrm{~s}$, the total power drops down to $2.5 \mathrm{MW}$.

\section{Acknowledgement}

This work has been partially supported by the ANR project TORID contract number ANR-12-BS03-0008.

\section{Appendix A. Estimation of the safety factor profile}

While the poloidal magnetic flux $\Psi_{r}$ is a natural choice to describe the plasma state, control objectives are generally formulated in terms of the safety factor $[6,43]$ or its inverse [14]. A change of variable is then necessary to convert $\Psi_{r}$ into the controlled variable. For example, the relation between $\iota(x, t)$ and $\Psi_{r}(x, t)$ can be written as in [13]:

$$
\begin{aligned}
\iota(x, t) & =-\left(\frac{\partial \Psi_{r}(x, t)}{\partial x}\right) \cdot\left(\frac{\partial x}{\partial \Phi(x, t)}\right) \\
& =-\frac{1}{2 \Phi_{\max }(t)}\left(\frac{1}{x} \frac{\partial \Psi_{r}(x, t)}{\partial x}\right)
\end{aligned}
$$

where the normalized radius is defined as $x=$ $\left(\Phi / \Phi_{\max }\right)^{1 / 2}$ and $\Phi_{\max }(t)=\Phi(1, t)$ is the toroidal flux inside the magnetic separatrix. The inverse of the safety factor can be presented by finite expansions on a different set of basis functions [14]. The approximation of $\iota$ with the basis function $\alpha_{k}(x)=(1 / x)\left(d a_{k} / d x\right)$, where $a_{k}$ are the cubic splines for $\Psi_{r}$ defined in Section 2, is

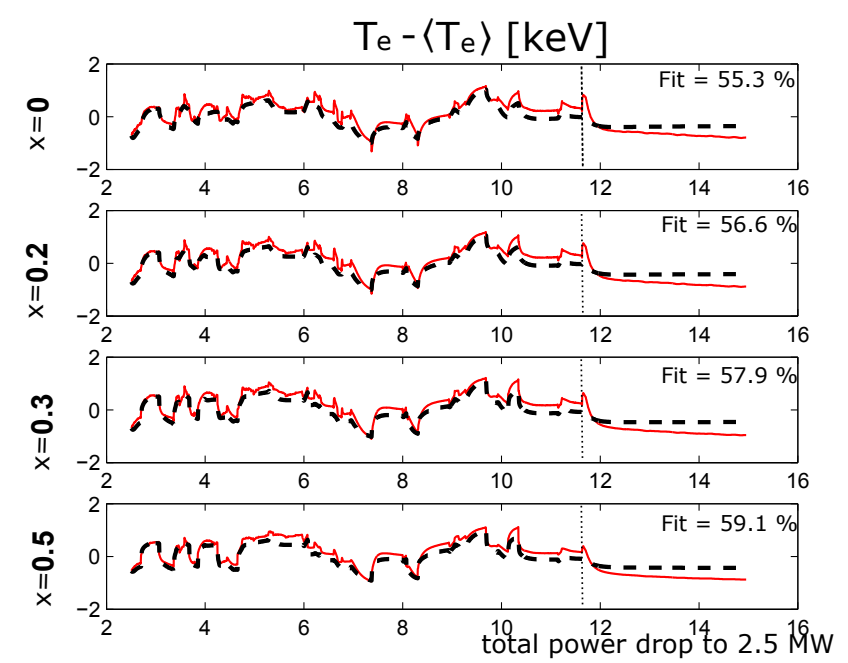

Figure 13: Plot of $T_{e}(t)$ vs time for the simulation \# 20 (see Table 1), The black dashed traces represent the outputs of the simulation of the identified system and the red traces represent the outputs of the original METIS simulation. The fit parameter defined in Eq. (13) is indicated in each frame. In this simulation at $11.6 \mathrm{~s}$, the total power drops down to $2.5 \mathrm{MW}$.

obtained as

$$
\iota(x, t)=-\frac{1}{2 \Phi_{\max }(t)} \sum_{k=1}^{n} \alpha_{k}(x) \Psi_{r k}(t)
$$

If $\Phi_{\max }$ is assumed to be constant, which is a good approximation when the toroidal field and the plasma shape are fixed, a matrix $C_{\iota}$ for the relation between $\iota(t)$ and $\Psi(t)$ can be found as:

$$
\tilde{\iota}(t)=C_{\iota} \cdot \tilde{X}=\left[\begin{array}{ll}
C_{\iota, \Psi_{r}} & 0
\end{array}\right]\left[\begin{array}{c}
\tilde{\Psi}_{r k}(t) \\
\tilde{T}_{e k}(t)
\end{array}\right]
$$

Once the model for the dynamics of $\Psi_{r}$ and $T_{e}$ is identified, the relation between $\iota$ and the states is approximated by (A.3) using the expression (A.2) to define the matrix $C_{\iota, \Psi_{r}}$.

\section{References}

[1] J. Wesson, D. Campbell, Tokamaks, Vol. 149, Oxford University Press, 2011.

[2] M. D. Boyer, J. Barton, E. Schuster, T. C. Luce, J. R. Ferron, M. L. Walker, D. A. Humphreys, B. G. Penaflor, R. D. Johnson, First-principles-driven model-based current profile control for the DIII-D tokamak via LQI optimal control, Plasma Physics and Controlled Fusion 55 (10) (2013) 105007.

[3] M. D. Boyer, J. Barton, E. Schuster, M. L. Walker, T. C. Luce, J. R. Ferron, B. G. Penaflor, R. D. Johnson, D. A. Humphreys, Backstepping control of the toroidal plasma current profile in the 
DIII-D tokamak, IEEE Transactions on Control Systems Technology 22 (5) (2014) 1725-1739.

[4] L. Laborde, D. Mazon, D. Moreau, A. Murari, R. Felton, L. Zabeo, R. Albanese, M. Ariola, J. Bucalossi, F. Crisanti, et al., A model-based technique for integrated real-time profile control in the JET tokamak, Plasma Physics and Controlled Fusion 47 (1) (2005) 155.

[5] N. M. T. Vu, R. Nouailletas, L. Lefèvre, F. Felici, Plasma q-profile control in tokamaks using a damping assignment passivity-based approach, Control Engineering Practice 54 (2016) 34-45.

[6] F. Bribiesca Argomedo, E. Witrant, C. Prieur, S. Brémond, R. Nouailletas, J. F. Artaud, Lyapunov-based distributed control of the safety factor profile in a tokamak plasma, Nuclear Fusion 53 (2013) 033005.

[7] F. Bribiesca Argomedo, C. Prieur, E. Witrant, S. Brémond, A strict control Lyapunov function for a diffusion equation with time-varying distributed coefficients, IEEE Transactions on Automatic Control 58 (2) (2013) 290-303.

[8] O. Gaye, E. Moulay, S. Brémond, L. Autrique, R. Nouailletas, J. Artaud, Y. Orlov, Robust stabilization of the current profile in tokamak plasmas using sliding mode approach in infinite dimension, Control Engineering Practice 21 (10) (2013) 1350-1358.

[9] O. Gaye, L. Autrique, Y. Orlov, E. Moulay, S. BréMond, R. Nouailletas, $h \infty$ stabilization of the current profile in tokamak plasmas via an LMI approach, Automatica 49 (9) (2013) 2795-2804.

[10] S. Mechhoud, E. Witrant, L. Dugard, D. Moreau, Estimation of heat source term and thermal diffusion in tokamak plasmas using a Kalman filtering method in the early lumping approach, IEEE Transactions on Control Systems Technology 23 (2) (2015) 449-463.

[11] E. Witrant, S. Brémond, Shape identification for distributed parameter systems and temperature profiles in tokamaks, in: 50th Conference on Decision and Control and European Control Conference, Orlando (FL),USA, 2011, pp. 2626-2631.

[12] D. Moreau, D. Mazon, M. Ariola, G. De Tommasi, L. Laborde, F. Piccolo, F. Sartori, T. Tala, L. Zabeo, A. Boboc, et al., A twotime-scale dynamic-model approach for magnetic and kinetic profile control in advanced tokamak scenarios on JET, Nuclear Fusion 48 (10) (2008) 106001.

[13] D. Moreau, D. Mazon, M. Walker, J. Ferron, K. Burrell, S. Flanagan, P. Gohil, R. Groebner, A. Hyatt, R. La Haye, et al., Plasma models for real-time control of advanced tokamak scenarios, Nuclear Fusion 51 (6) (2011) 063009.

[14] D. Moreau, M. L. Walker, J. R. Ferron, F. Liu, E. Schuster, J. E. Barton, M. D. Boyer, K. H. Burrell, S. Flanagan, P. Gohil, et al., Integrated magnetic and kinetic control of advanced tokamak plasmas on DIII-D based on data-driven models, Nuclear Fusion 53 (6) (2013) 063020

[15] S. Kim, J. Lister, A potentially robust plasma profile control approach for iter using real-time estimation of linearized profile response models, Nuclear Fusion 52 (7) (2012) 074002.

[16] J. E. Barton, W. P. Wehner, E. Schuster, F. Felici, O. Sauter, Simultaneous closed-loop control of the current profile and the electron temperature profile in the TCV tokamak, in: American Control Conference, Chicago, USA, 2015, pp. 3316-3321.

[17] J. E. Barton, K. Besseghir, J. Lister, E. Schuster, Physicsbased control-oriented modeling and robust feedback control of the plasma safety factor profile and stored energy dynamics in ITER, Plasma Physics and Controlled Fusion 57 (11) (2015) 115003.

[18] J. Barton, M. Boyer, W. Shi, W. Wehner, E. Schuster, J. Ferron, M. Walker, D. Humphreys, T. Luce, F. Turco, et al., Physics-model-based nonlinear actuator trajectory optimization and safety factor profile feedback control for advanced scenario development in DIII-D, Nuclear Fusion 55 (9) (2015) 093005.

[19] V. Basiuk, J. Artaud, F. Imbeaux, X. Litaudon, A. Bécoulet, L.G. Eriksson, G. Hoang, G. Huysmans, D. Mazon, D. Moreau, et al., Simulations of steady-state scenarios for tore supra using the CRONOS code, Nuclear Fusion 43 (9) (2003) 822.

[20] J. Artaud, V. Basiuk, F. Imbeaux, M. Schneider, J. Garcia, G. Giruzzi, P. Huynh, T. Aniel, F. Albajar, J. Ane, et al., The CRONOS suite of codes for integrated tokamak modelling, Nuclear Fusion 50 (4) (2010) 043001.

[21] F. Felici, O. Sauter, S. Coda, B. Duval, T. Goodman, J. Moret, J. Paley, T. Team, et al., Real-time physics-model-based simulation of the current density profile in tokamak plasmas, Nuclear Fusion 51 (8) (2011) 083052.

[22] F. Felici, O. Sauter, Non-linear model-based optimization of actuator trajectories for tokamak plasma profile control, Plasma Physics and Controlled Fusion 54 (2) (2012) 025002.

[23] G. Pereverzev, P. Yushmanov, ASTRA automated system for transport analysis in a tokamak report ipp 5/98, Max-PlanckInstitut für Plasmaphysik, Garching (2002).

[24] P. Geelen, F. Felici, A. Merle, O. Sauter, Parameter estimation for a nonlinear control-oriented tokamak profile evolution model, Plasma Physics and Controlled Fusion 57 (12) (2015) 125008.

[25] T. Katayama, Subspace methods for system identification, Springer Science \& Business Media, 2006.

[26] L. Ljung, System identification, Springer, 1998.

[27] M. Verhaegen, P. Dewilde, Subspace model identification part 1. the output-error state-space model identification class of algorithms, International Journal of Control 56 (5) (1992) 11871210.

[28] D. Di Ruscio, Subspace system identification: Theory and applications, Lecture notes, Telemark University College, Porsgrunn, Norway.

[29] B. Haverkamp, Subspace method identification, theory and practice, Ph.D. thesis, Ph. D. thesis, TU Delft, Delft, The Netherlands (2000).

[30] M. Verhaegen, V. Verdult, Filtering and system identification: a least squares approach, Cambridge university press, 2007.

[31] W. Suleiman, A. Monin, Linear multivariable system identification: multi-experiments case, in: Conference on Systems and Control, Marrakech, Morocco, 2007.

[32] J. Blum, Numerical Simulation and Optimal Control in Plasma Physics: With Applications to Tokamaks (Wiley GauthierVillars Series in Modern Applied Mathemat), 1989.

[33] E. Witrant, E. Joffrin, S. Brémond, G. Giruzzi, D. Mazon, O. Barana, P. Moreau, A control-oriented model of the current profile in tokamak plasma, Plasma Physics and Controlled Fusion 49 (7) (2007) 1075.

[34] F. Hinton, R. Hazeltine, Theory of plasma transport in toroidal confinement systems, Reviews of Modern Physics 48 (2) (1976) 239.

[35] J. Shen, Efficient spectral-Galerkin methods III: Polar and cylindrical geometries, SIAM Journal on Scientific Computing 18 (6) (1997) 1583-1604.

[36] D. Bauer, On data preprocessing for subspace methods, in: 39th IEEE Conference on Decision and Control, Vol. 3, Sydney, Australia, 2000, pp. 2403-2408.

[37] L. Duchesne, E. Feron, J. D. Paduano, M. Brenner, Subspace identification with multiple data sets, in: Proceedings of the 1996 AIAA Guidance, Navigation, and Control Conference, San Diego (CA), USA, 1996.

[38] J. Ramos, Hermitian operator methods for reaction-diffusion equations, Numerical Methods for Partial Differential Equations 3 (4) (1987) 241-287. 
[39] D. N. Miller, R. A. De Callafon, Subspace identification with eigenvalue constraints, Automatica 49 (8) (2013) 2468-2473.

[40] M. Chilali, P. Gahinet, Ho design with pole placement constraints: an LMI approach, IEEE Transactions on Automatic Control 41 (3) (1996) 358-367.

[41] J. Löfberg, YALMIP: A toolbox for modeling and optimization in matlab, in: IEEE International Symposium on Computer Aided Control Systems Design, Taipei, Taiwan, 2004, pp. 284289.

[42] D. Leith, D. Murray-Smith, R. Bradley, Combination of data sets for system identification, in: IEE Proceedings D-Control Theory and Applications, Vol. 140, IET, 1993, pp. 11-18.

[43] J. E. Barton, K. Besseghir, J. Lister, E. Schuster, Robust control of the safety factor profile and stored energy evolutions in high performance burning plasma scenarios in the ITER tokamak, in: 52nd Conference on Decision and Control, Florence, Italy, 2013, pp. 4194-4199. 